\title{
Photon scanning tunneling microscope in combination with a force microscope
}

\author{
M. H. P. Moers, R. G. Tack, N. F. van Hulst, and B. Bölger \\ Department of Applied Physics, University of Twente, P. O. Box 217, 7500 AE Enschede, \\ The Netherlands
}

(Received 24 June 1993; accepted for publication 19 October 1993)

\begin{abstract}
The simultaneous operation of a photon scanning tunneling microscope with an atomic force microscope is presented. The use of standard atomic force silicon nitride cantilevers as near-field optical probes offers the possibility to combine the two methods. Vertical forces and torsion are detected simultaneously with the optical near field, which allows a comparison between topography and the optical signal. Images of an optical thin film (indium tin oxide) and a Langmuir-Blodgett layer (pentacosa diynoic acid) show absorption contrast with a lateral resolution of about $30 \mathrm{~nm}$ (based on edge steepness), which is well below the diffraction limit.
\end{abstract}

\section{INTRODUCTION}

In contrast to the optical far field, as detected by lensebased microscopes, the field in the direct proximity of an illuminated object yields information about the detailed structure of the object, not limited by diffraction. Detection of the optical field near the object is the base of a class of microscopes gathered under the name scanning near-field optical microscopy (SNOM).

Although the idea of superresolution in the near field had already been proposed by Synge, ${ }^{1}$ and experimentally verified in the microwave regime by Ash and Nicholls, ${ }^{2}$ the first near-field microscopes working at optical wavelengths were built in the 1980s. ${ }^{3,4}$ In these microscopes an aperture, much smaller than the optical wavelength, is raster scanned over a surface, working as a point source and/or detector. Apart from the fabrication of the aperture, the main problem was the distance regulation, necessary to operate the probe in the near field. Nowadays, resolutions down to $10 \mathrm{~nm}$ have been shown with aluminum-coated adiabatically tapered optical fibers and feedback on shear force. ${ }^{5}$ One drawback of these coated fibers is the outer diameter of about $200 \mathrm{~nm}$, which limits the operation to extremely fiat surfaces.

Another approach, referred to as photon scanning tunneling microscopy (PSTM), ${ }^{6-9}$ is based on the local detection of the electromagnetic field near the sample when it is illuminated with a laser beam under total internal reflection. A sample placed on the substrate generates field distributions that contain information about both topological and optical properties of the sample. When imaging with far-field techniques, i.e., lenses, this method resembles dark-field illumination, for only scattered light is detected. ${ }^{10}$ The field close to the surface, however, gives more detailed information about the sample. A small dielectric probe placed in this near field is able to convert the local field into a propagating wave, thus working as a nearfield detector. ${ }^{11,12}$ A naive representation of the principle is that of a small scatterer in an electromagnetic field. More profound theories take into account the total probe-sample interaction. $^{13}$

Like in all scanning probe microscopes, the lateral res- olution in PSTM is mainly determined by the locality of the interaction. Here, the dimensions and geometry of the probe and the distance between probe and sample are important parameters. The size and geometry of the probe affect the local coupling of the field into the probe and the way the light is guided toward the detector. Because subwavelength information about the structure is lost in the far field, the probe should be operated within a few nanometers from the sample.

Traditionally, chemically sharpened optical fibers are used as near-field detectors. ${ }^{6-8}$ Tip diameters of about 100 $\mathrm{nm}$ are easily obtained, though much smaller probes are hard to produce. One should notice that, due to the rigidity of the fiber along the axis, a single touch of the sample usually destroys the probe. Therefore a proper distance regulation is essential to avoid tip damage. In order to operate the probe in the near field, a feedback on constant optical intensity is commonly used. However, although this method appears to work on bare glass and on simple highly symmetric structures, most samples generate complex field distributions, on which a distance control based on intensity does not work. The variations in intensity remove the probe too far from the surface or cause a fatal touch of the surface.

Probably the best alternative for distance regulation is a feedback on force. In a previous paper, ${ }^{14}$ an instrument has been presented in which silicon nitride cantilevers with integrated pryamidal tip, ${ }^{15}$ commercially available for atomic force microscopy (AFM) ${ }^{16}$ are used as near-field probes. These probes are suitable for PSTM for several reasons. First, silicon nitride is optically transparent down to $300 \mathrm{~nm}$ with $n=2.0$. Second, the cantilevers are microfabricated with a tip radius of $30 \mathrm{~nm}$. And, last but not least, the cantilevers have a low force constant $(0.1 \mathrm{~N} / \mathrm{m})$, which avoids tip damage when touching the surface.

In spite of these advantageous properties the silicon nitride probes have some drawbacks: the large apex and the geometry of cantilever. The light is not guided to the detector like with fiber tips, but has to be collected by a lens.

In the present paper, a combination of this instrument with a force microscope is presented. An optical beam de- 


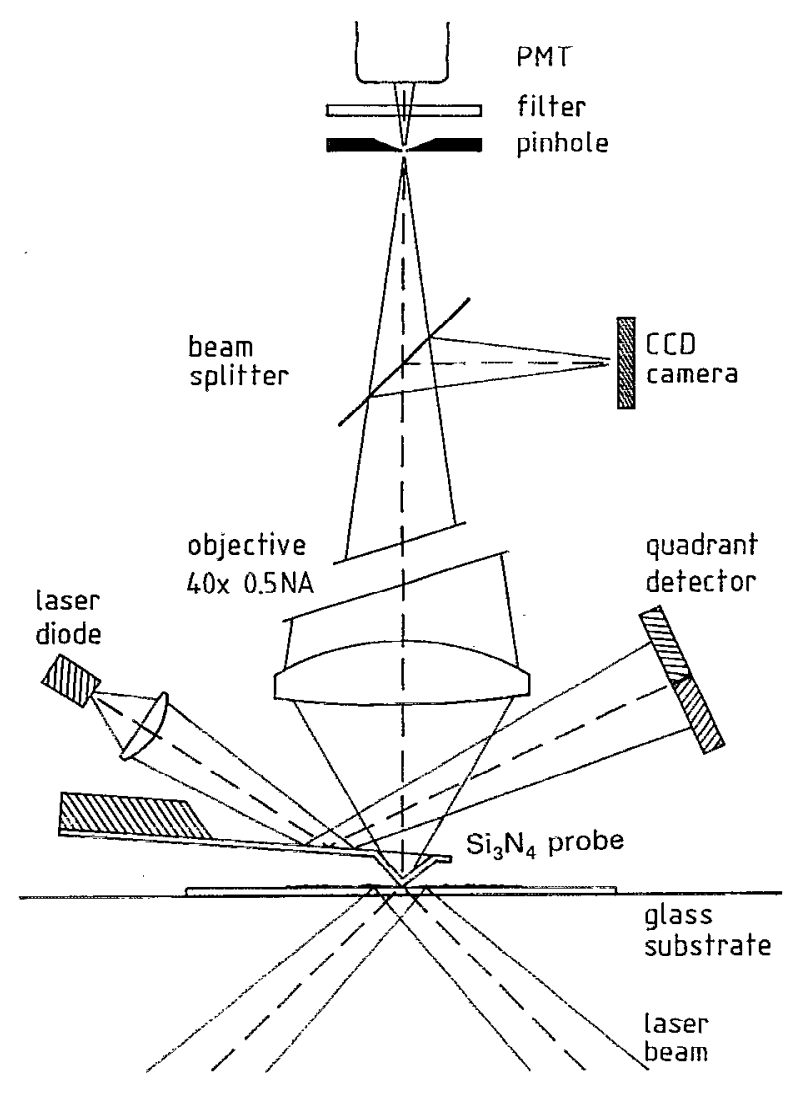

FIG. 1. The combination of PSTM (tip-objective-PMT), AFM (laser diode-cantilever-quadrant detector) and conventional microscope (beam splitter-CCD camera). The beam deflection system is oriented perpendicular to the paper, but is drawn in plane for clarity.

flection system ${ }^{17}$ is included that makes it possible to operate the instrument as a PSTM and an AFM simultaneously. This offers the opportunity to compare the PSTM images with the topography of the sample. In the following sections the combined PSTM-AFM setup will be described. Some results of scans on an optical thin film (indium tin oxide) and an absorbing Langmuir-Blodgett layer will be discussed.

\section{THE INSTRUMENT}

In the instrument the sample is placed on a glass substrate (BK7) with index matching oil and illuminated under total internal reflection by an argon ion laser beam (Fig. 1). The optical power in these experiments typically is a few milliwatts weakly focused $(\mathrm{NA}=0.02)$ to a spot of $50 \mu \mathrm{m}$. The total internal reflection creates an evanescent field in the low index medium, which is modified by the sample. In the far field, scattered light is detected with diffraction limited resolution. In the near field, however, more information about the subwavelength structure of the sample is present. The silicon nitride tip, placed on the sample, partially converts the near field into a radiative field. This is clearly seen by a bright spot at the tip position. The gold coating, present on the cantilevers (Park Scientific Instruments), has been removed by etching in a $\mathrm{HCl}-\mathrm{HNO}_{3}$ (2:1) solution (King's water). The light, gen- erated at the tip, is collected with a long working distance objective $(40 \times, \mathrm{NA}=0.5,10 \mathrm{~mm})$. The light from the tip is selected by a $100 \mu \mathrm{m}$ pinhole in the image plane and detected by a photo multiplier tube (PMT). A BG39 colored glass filter is placed in the optical path to avoid the detection of scattered infrared light used in the atomic force part of the microscope. The signal level typically is $0.1 \mathrm{nW}$. By scanning the sample with a three-axis piezopositioner (Photon Control) a PSTM image is obtained.

An AFM is incorporated in the instrument by means of a beam deflection system. A diode laser $(\lambda=780 \mathrm{~nm})$ is focused on the cantilever and the displacement of the reflected beam, caused by deflection of the cantilever, is detected with a quadrant detector. Because of the limited space between the cantilever holder and the high NA objective, the beam deflection system is oriented in a plane perpendicular to the long axis of the cantilever.

The AFM can be operated either in constant height mode or in constant force mode. ${ }^{17}$ While scanning in constant force mode, the sample is adjusted in such a way that the deflection of the cantilever is kept zero, indicating no force between probe and sample.

A personal computer is used to generate the scan pattern and to collect the data. The images shown in this paper are $300 \times 300$ pixels in size. Per pixel, the PSTM signal and up to three AFM signals are measured. In constant height mode the deflection and the torsion of the cantilever are monitored. Torsion of the cantilever is due to forces on the tip parallel to the sample. When scanning in constant force mode the piezovoltage necessary to compensate for the height, the error signal (due to the limited bandwidth of the feedback loop), and the torsion of the cantilever are detected. Furthermore, it is possible to choose a scan direction parallel or perpendicular to the symmetry axis of the cantilever (the latter giving a friction force image). ${ }^{18}$

In addition to these two scanning probe microscopes, a conventional optical microscope is included by means of a beam splitter and a CCD camera. The conventional microscope is essential for selecting the object of interest.

\section{RESULTS AND DISCUSSION}

The operation of a photon scanning tunneling microscope is best demonstrated on samples that display little topography on a wavelength scale, such that the far-field contributions will not dominate over the near-field contributions. For this reason an optical thin film of indium tin oxide (ITO, Baltracon) is a suitable sample. ITO has a granular structure with crystallites of 50-200 nm in size. In this experiment $p$-polarized light of $514 \mathrm{~nm}$ is used. Figures 2 (a) -2 (d) show the results of a $3.7 \times 3.7 \mu \mathrm{m}$ scan on ITO.

The structure of ITO is clearly resolved in the force images [Figs. 2(a)-2(c)], giving piezovoltage, error signal, and torsion signal, respectively. The resolution of the AFM is limited to $10-50 \mathrm{~nm}$ by tip convolution. Figure 2(d) shows the simultaneously produced PSTM image, in which the grains show dark due to their higher refractive index and limited transmittance. The resolution in the optical image is comparable with the AFM image. 


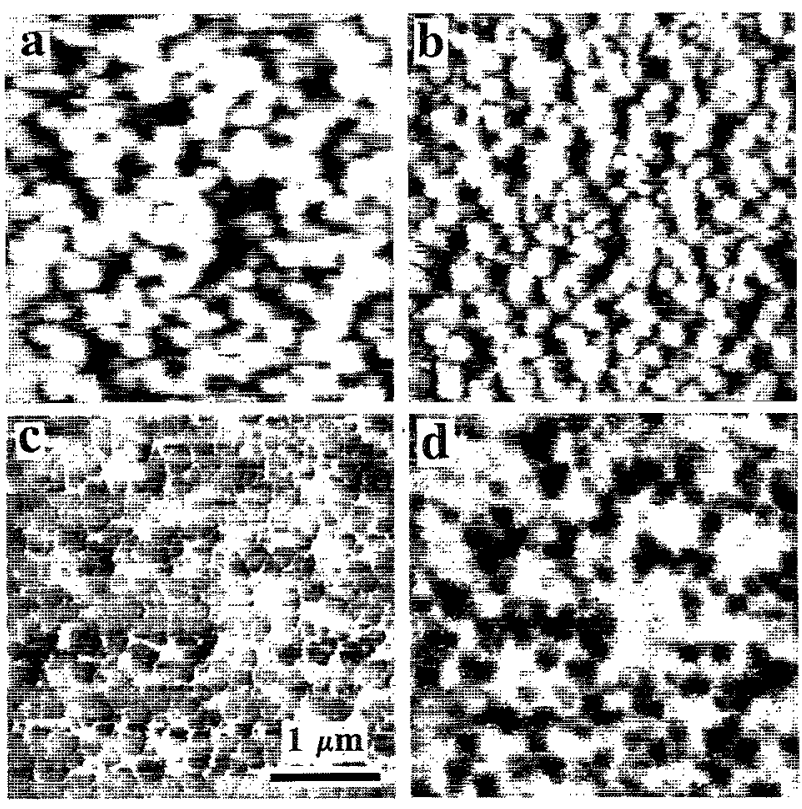

FIG. 2. A $3.7 \times 3.7 \mu \mathrm{m}$ scan of indium tin oxide: (a) piezovoltage (height), (b) error signal, (c) torsion signal, and (d) PSTM.

This scan is made in constant force mode. However, no differences are found when scanning in constant height mode, where the force is not kept constant. Therefore we can conclude that the scanning force does not influence the optical signal.

In order to investigate the spectroscopic capabilities of the instrument, several scans have been made on polymerized Langmuir-Blodgett films of 10,12-pentacosadiynoic acid (PCA) ${ }^{19-22}$ These PCA films form domains on the substrate with lateral dimensions of a fraction of a micron to several microns, and a height of only $6 \mathrm{~nm}$. They show strong absorption bands at 505 and $555 \mathrm{~nm}$ and emission bands at 562 and $640 \mathrm{~nm}\left(\lambda_{\mathrm{ex}}=514 \mathrm{~nm}\right)$. The absorption in each domain is strongly dependent on the polarization direction of the excitation light because the absorption dipole moment is parallel to the polycarbon backbone, which is highly oriented.

In the first experiments on these LB films the incoming beam is $p$-polarized and $\lambda=514 \mathrm{~nm}$. A UG9 filter between
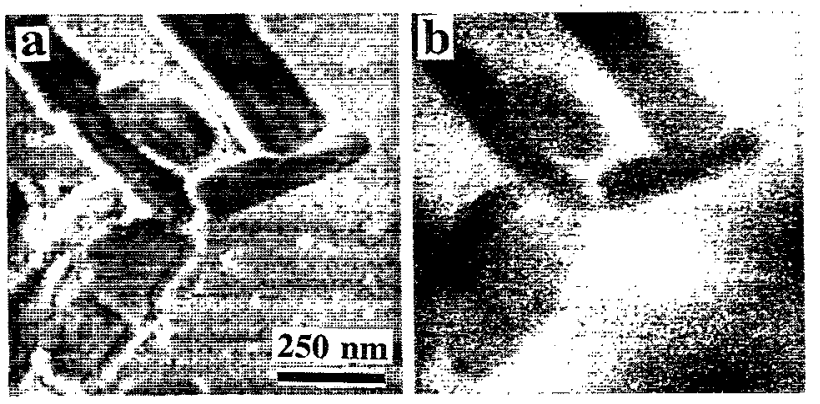

FIG. 3. A $1 \times 1 \mu \mathrm{m}$ scan of a PCA film showing (a) the AFM friction image and (b) the corresponding PSTM image.

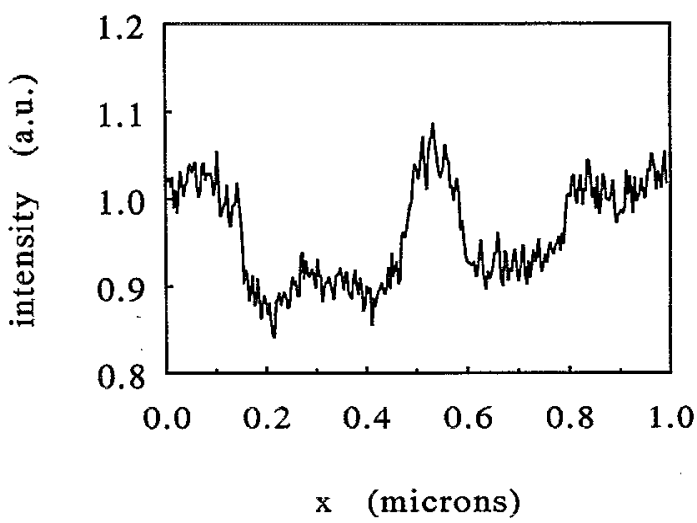

FIG. 4. A line profile taken from Fig. 3(b) showing the intensity difference between the substrate and the LB film.

objective and pinhole selects the green light, such that only the absorption of the film is measured. Figure 3 shows a $1 \times 1 \mu \mathrm{m}$ scan of several domains on the glass substrate. The friction image [Fig. 3(a)] and the PSTM image [Fig. 3 (b)] are line-equalized in order to remove some very lowfrequency variations in intensity. The height and error signal are not shown because they show low contrast.

Domains of the LB film are clearly visible in the PSTM image with a resolution of about $30 \mathrm{~nm}$ (based on edge steepness), which is well below the wavelength of the incoming light. The contrast is believed to come from absorption of the light by the film. The intensity difference between the LB film and the substrate, as seen in a line scan (Fig. 4), is about 10\%. This is in agreement with the absorption of the film at $514 \mathrm{~nm}$, i.e., $7 \%$, where we have to take into account the fact that only about half of substrate is covered with LB film, such that the local absorption is higher than the macroscopic absorption. This agreement indicates a contrast due to the optical properties of the PCA film.

\section{CONCLUSION}

The operation of a PSTM in combination with an AFM has been demonstrated in this paper. The use of silicon nitride cantilevers as near-field probes instead of the traditional etched optical fibers shows several advantages. Because the tip is placed on a thin cantilever, repeated close contact scanning is possible without tip destruction. The results in this paper have been reproduced several times with different probes. The integration of the AFM in the instrument is a great advantage, for it offers the opportunity to compare the PSTM image with the topography of the structure under investigation. The measurements on indium tin oxide and PCA films show that high-resolution imaging is possible. Future work will be aimed at showing polarization contrast on these LB films.

\section{ACKNOWLEDGMENTS}

The authors wish to thank F. B. Segerink, K. O. van der Werf, and E. H. Schipper for their help with the con- 
struction of hard- and software. We thank H. E. Gaub for providing the Langmuir-Blodgett films. This work is mainly supported by the Dutch Foundation for Fundamental Research on Matter (FOM).

${ }^{I}$ E. H. Synge, Philos. Mag. 6, 356 (1928).

${ }^{2}$ E. A. Ash and G. Nicholls, Nature 237, 510 (1972).

${ }^{3}$ D. W. Pohl, W. Denk, and M. Lanz, Appl. Phys. Lett. 44, 651 (1984).

${ }^{4}$ A. Harootunian, E. Betzig, M. Isaacson, and A. Lewis, Appl. Phys. Lett. 49, 674 (1986).

${ }^{5}$ E. Betrig and J. K. Trautman, Science 257, 189 (1992).

${ }^{6}$ C. Reddick, R. J. Warmack, and T. L. Ferrel, Phys. Rev. B 39, 767 (1989).

${ }^{7}$ D. Courjon, K. Sarayeddine, and M. Spajer, Opt. Commun. 71, 23 (1989).

${ }^{8}$ N. F. van Hulst, N. P. de Boer, and B. Bölger, J. Microsc. 163, 117 (1991).

${ }^{9}$ N. F. van Hulst, F. B. Segerink, F. Achten, and B. Bölger, Ultramicroscopy 42-44, 416 (1992).

${ }^{10} \mathrm{~J}$. M. Murray and D. Eshel, J. Microsc. 167, 49 (1992).

IIJ. M. Vigoureux, C. Girard, and D. Courjon, Opt. Lett. 14, 1039 (1989).
${ }^{12}$ D. Courjon, C. Bainier, C. Girard, and J. M. Vigoureux, Ann. Phys. 2, 149 (1993).

${ }^{13}$ C. Girard and X. Bouju, J. Chem. Phys. 96, 2056 (1991).

${ }^{14}$ N. F. van Hulst, M. H. P. Moers, O. F. J. Noordman, R. G Tack, F. B. Segerink, and B. Bölger, Appl. Phys. Lett. 62, 461 (1993).

${ }^{15}$ T. R. Albrecht, S. Akamine, T. E. Carver, and C. F. Quate, J. Vac. Sci. Technol. A 8, 3386 (1990).

${ }^{16}$ G. Binnig, C. F. Quate, and Ch. Gerber, Phys. Rev. Lett. 56, 930 (1986).

${ }^{17}$ G. Meyer and N. M. Amer. Appl. Phys. Lett. 53; 2400 (1988).

${ }^{18}$ R. M. Overney, E. Meyer, J. Frommer, D. Brodbeck, R. Lüthi, L. Howald, H. J. Güntherodt, M. Fujihira, H. Takano, and Y. Gotoh, Nature 359, 133 (1992).

${ }^{19}$ B. M. Gocttgens, R. W. Tillmann, M. Radmacher, and H. E. Gaub, Langmuir 8, 1768 (1992).

${ }^{20}$ C. A. J. Putman, H. G. Hansma, H. E. Gaub, and P. K. Hansma, Langmuir 8, 3014 (1992).

${ }^{21}$ H. D. Göbel, H. E. Gaub, and H. Möhwald, Chem. Phys. Lett. 138, 441 (1987).

${ }^{22}$ C. Bubeck, B. Tieke, and G. Wegner, Ber. Bunsenges. Phys. Chem. 86, 495 (1982). 